\title{
Antitumor effects of anlotinib in thyroid cancer
}

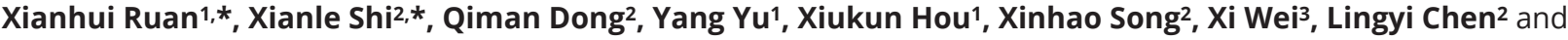 \\ Ming Gao' \\ 1Department of Thyroid and Neck Tumor, Tianjin Medical University Cancer Institute and Hospital, National Clinical Research Center for Cancer, \\ Key Laboratory of Cancer Prevention and Therapy, Tianjin, China \\ ${ }^{2}$ State Key Laboratory of Medicinal Chemical Biology, Key Laboratory of Bioactive Materials, Ministry of Education, Collaborative Innovation Center for \\ Biotherapy, Tianjin Key Laboratory of Protein Sciences, 2011 Collaborative Innovation Center of Tianjin for Medical Epigenetics and College of Life \\ Sciences, Nankai University, Tianjin, China \\ 3Department of Diagnostic and Therapeutic Ultrasonography, Tianjin Medical University Cancer Institute and Hospital, National Clinical Research Center \\ of Cancer, Key Laboratory of Cancer Prevention and Therapy, Tianjin, China
}

Correspondence should be addressed to L Chen or M Gao: lingyichen@nankai.edu.cn or gaoming68@aliyun.com

*(X Ruan and X Shi contributed equally to this work)

\begin{abstract}
There is no effective treatment for patients with poorly differentiated papillary thyroid cancer or anaplastic thyroid cancer (ATC). Anlotinib, a multi-kinase inhibitor, has already shown antitumor effects in various types of carcinoma in a phase I clinical trial. In this study, we aimed to better understand the effect and efficacy of anlotinib against thyroid carcinoma cells in vitro and in vivo. We found that anlotinib inhibits the cell viability of papillary thyroid cancer and ATC cell lines, likely due to abnormal spindle assembly, G2/M arrest, and activation of TP53 upon anlotinib treatment. Moreover, anlotinib suppresses the migration of thyroid cancer cells in vitro and the growth of xenograft thyroid tumors in mice. Our data demonstrate that anlotinib has significant anticancer activity in thyroid cancer, and potentially offers an effective therapeutic strategy for patients of advanced thyroid cancer type.
\end{abstract}

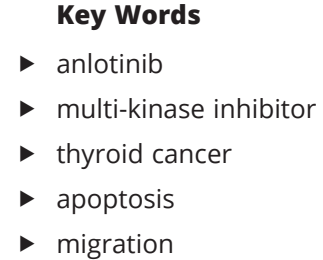

Endocrine-Related Cancer (2019) 26, 153-164

\section{Introduction}

Thyroid cancer is the most common cancer of endocrine system, with a rapid worldwide increase over recent decades (Enewold et al. 2009, Kilfoy et al. 2009, Chen et al. 2016). The disease is classified into three types based on pathological characteristics: papillary carcinoma (PTC), follicular carcinoma (FTC) and anaplastic carcinoma (ATC) (Cho et al. 2013). About 90\% of thyroid cancer are well differentiated, while $10 \%$ or less are poorly differentiated or anaplastic subtypes (Kondo et al. 2006, Xing 2013). Of the differentiated carcinomas, 85-90\% are PTC and 10-15\% are FTC (Baudin \& Schlumberger 2007). ATC is a rare, but very aggressive, human malignant tumor. The approximate incidence of ATC is one to two cases per million every year, but the median survival of
ATC patients is only about five months (Nagaiah et al. 2011, Smallridge et al. 2012). Most thyroid cancer patients become disease-free after initial treatment with surgical resection, radioiodine, and thyroid hormone therapy (McFarland \& Misiukiewicz 2014). However, there are few treatment options available for patients with advanced disease, including radioiodine-resistant and metastatic differentiated thyroid cancer and anaplastic thyroid cancer (ATC). Tumors initially categorized as poorly differentiated thyroid cancer (PDTC) or ATC are often highly aggressive and recurrent. In addition to their aggressive growth and metastasis, loss of the capacity to uptake iodine makes both PDTC and ATC difficult to treat, leading to poor prognosis (Smallridge et al. 
2009, McFarland \& Misiukiewicz 2014). Moreover, chemotherapeutic treatment has been proved to be largely ineffective against aggressive thyroid carcinomas. These inadequacies of current treatment protocols for PDTC and ATC strongly emphasize the urgent need for novel targeted treatment options (Sherman 2009).

Over the past few decades, significant advances have been made in the understanding of the molecular pathogenesis of thyroid cancer (Xing 2013). The pathogenesis of thyroid cancer is thought to involve a multi-step process, in which genetic alterations in oncogenes and tumor suppressor genes lead to aberrant proliferation of cells, and alterations in angiogenic genes lead to tumor invasion and spread (Fagin \& Mitsiades 2008). Some important tumorigenic factors have been identified as potential therapeutic targets for novel anticancer treatments. Multi-targeted tyrosine kinase inhibitors have demonstrated significant antitumor effects in a variety of tumor types, including thyroid cancer, by inhibiting the angiogenic and proliferative signaling (Lorusso et al. 2016). Recently, some kinase inhibitors such as sorafenib, vandetanib and cabozantinib have been proved to be the first-line therapies of advanced thyroid malignancies. In addition, more and more multi-kinase inhibitors are included in clinical trials (Covell \& Ganti 2015).

Anlotinib is a new multi-kinase inhibitor that has shown efficacy against a wide variety of tumors in preclinical models. It has been reported that anlotinib is safe and efficient to treat patients with advanced refractory solid tumors (Sun et al. 2016). Anlotinib suppresses tumor cell proliferation and angiogenesis, via inhibition of platelet-derived growth factor receptor, Ret, Aurora-B, epidermal growth factor receptor and fibroblast growth factor receptor (FGFR) (Wang et al. 2016).

The purpose of the studies reported here was to investigate the antitumor efficacy and mechanism of anlotinib in preclinical models of PTC and ATC. Three PTC cell lines and three ATC cell lines were used to elucidate the effects of anlotinib at different doses on proliferation. The $\mathrm{IC}_{50}$ of anlotinib on these cells range from 3.02 to $5.42 \mu \mathrm{M}$. We found that anlotinib inhibits the cell viability of thyroid cancer cells, and arrests cells at the G2/M phase, most likely due to abnormal spindle assembly, but not the BRAF/MEK/ERK pathway, one of the most important signaling pathways in thyroid cancer. Cell apoptosis assay revealed that anlotinib induces apoptosis of thyroid cancer cells, partly through activating the TP53 pathway. Anlotinib also inhibits the migration of thyroid cancer cells, through interfering F-actin formation. In addition, anlotinib suppresses the growth of xenograft thyroid tumors in mice. These data provided the first evidence that anlotinib may have a high therapeutic efficacy in thyroid cancer, as both antitumor and antimetastatic agents.

\section{Materials and methods}

\section{Compounds}

Anlotinib was kindly provided by Tai Tianqing (Nanjing, China). PD0325901 was purchased from Sigma. Compounds were dissolved in dimethyl sulfoxide (DMSO, Sigma), and diluted with culture medium to the desired concentration for in vitro studies.

\section{Cell lines and cell culture}

Three PTC cell lines, BCPAP, K1, and IHH4, and three ATC cell lines, 8505C, CAL-62, and BHT-101, were used in this study. $\mathrm{K} 1, \mathrm{IHH} 4$ and $8505 \mathrm{C}$ were kindly provided by Prof. Haixia Guan (China Medical University, China) and all other cell lines were purchased from American Type Culture Collection. BCPAP, K1, IHH4, and 8505C cells were cultured in growth medium consisting of 90\% RPMI-1640 (Gibco), 10\% FBS, 2 mM L-glutamine, 5000 units/mL penicillin and streptomycin. CAL-62 and BHT-101 cells were cultured in growth medium consisting of $85 \%$ DMEM (high glucose, Invitrogen), 15\% FBS, $2 \mathrm{mM}$ L-glutamine, 5000 units/mL penicillin and streptomycin. All the cell lines were authenticated with Short Tandem Repeat DNA profiling analysis routinely, BRAF mutational status of BCPAP, K1 and $8505 \mathrm{C}$ were verified by sanger sequencing. The passage number of the cells used for the experiments was about 20-30.

\section{Cell viability assay}

Cell viability was measured using MTT (Sangon, China). Cells $\left(5 \times 10^{3} /\right.$ well $)$ were seeded into 96 -well plate and incubated for $24 \mathrm{~h}$. Then $100 \mu \mathrm{L}$ medium containing 0 , $0.01,0.1,0.4,0.8,1,4,8,10,15$ and $20 \mu \mathrm{M}$ anlotinib were added into each well. After $72 \mathrm{~h}$ anlotinib treatment, cells were incubated with MTT substrates $(5 \mathrm{mg} / \mathrm{mL})$ for $4 \mathrm{~h}$. Culture medium was removed, and DMSO was added. Optical density was measured at $570 \mathrm{~nm}$. Calculation of results and Student's t-test were performed using SoftMax pro software (Molecular Devices), and $\mathrm{IC}_{50}$ values were calculated using Sigma Plot software (Systa, San Jose, CA, USA).

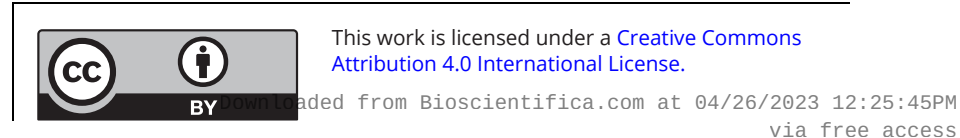




\section{Cell cycle analysis}

Cells were treated with DMSO or $10 \mu \mathrm{M}$ anlotonib for $24 \mathrm{~h}$. Treated cells were harvested, fixed in cold $70 \%$ ethanol, and incubated at $4^{\circ} \mathrm{C}$ overnight. Cells were resuspended in PBS buffer supplemented with $60 \mu \mathrm{g} / \mathrm{mL}$ RNase A and $25 \mathrm{\mu g} / \mathrm{mL}$ propidium iodide (PI), and incubated $20 \mathrm{~min}$ in dark at $37^{\circ} \mathrm{C}$. Samples were analyzed with a FACS Calibur flow cytometer (BD Biosciences).

\section{Cell apoptosis analysis}

Cells were treated with DMSO or $10 \mu \mathrm{M}$ anlotinib for $36 \mathrm{~h}$. Cells collected by trypsinization and washed with PBS. To detect cell death, an Annexin V apoptosis detection kit (Beyotime) was used. Briefly, $1 \times 10^{5}$ cells were mixed with Annexin V-FITC and PI, and incubated for $15 \mathrm{~min}$ at room temperature in the dark. Stained cells were analyzed with a FACS Calibur flow cytometer (BD Biosciences).

\section{Antibodies and Western blotting}

Cells were lysed in lysis buffer (Beyotime), and protein concentration was measured using BCA Protein Assay kit (Beyotime) to ensure equal loading. The samples were resolved by SDS-PAGE, followed by transferring onto a PVDF membrane (Millipore).

A

\section{Anlotinib}

$(\mu \mathrm{M})$ :

BCPAP

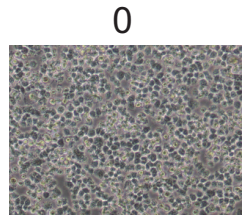

$8505 \mathrm{C}$

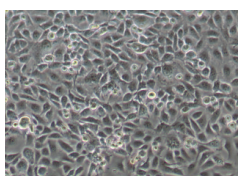

\section{1}
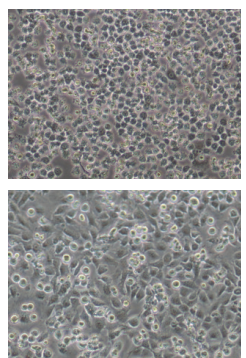

5
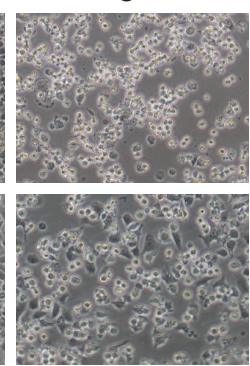
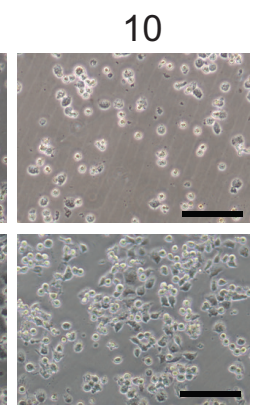

B
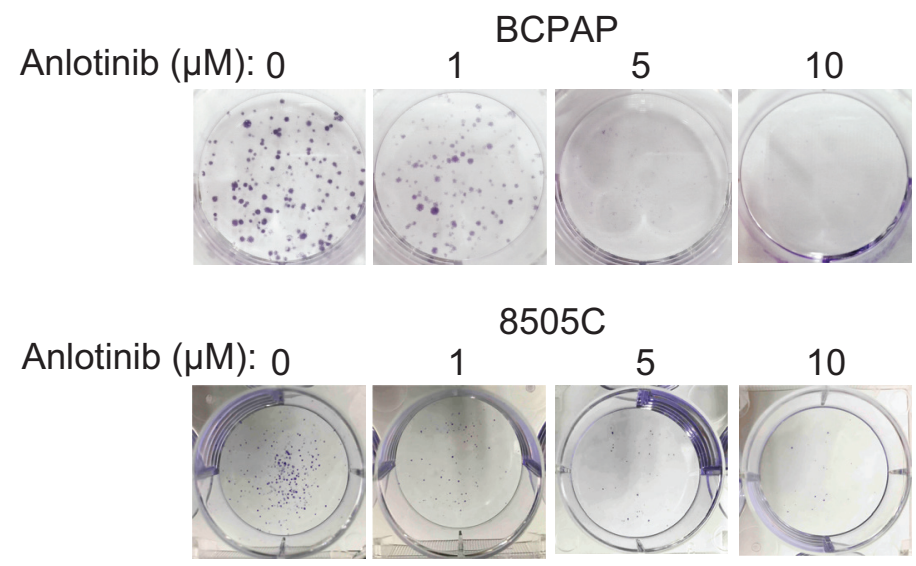

C

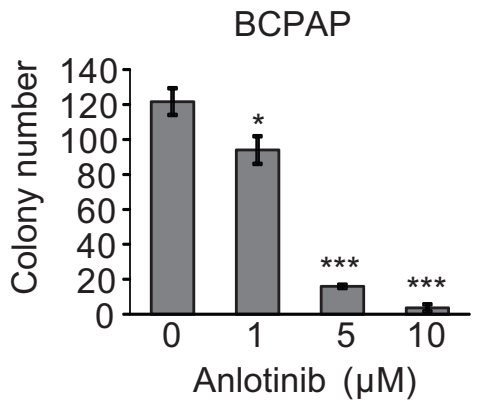

D

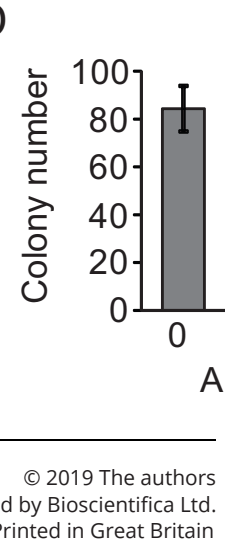

$8505 \mathrm{C}$

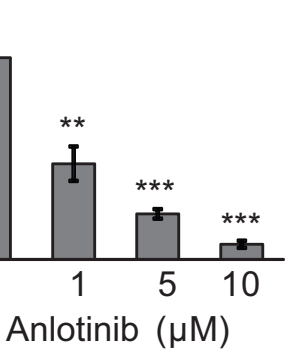

\section{Figure 1}

Anlotinib inhibits thyroid cancer cells proliferation in vitro. (A) Thyroid cancer cells BCPAP and $8505 \mathrm{C}$ were treated with anlotinib $(0,1,5$ and $10 \mu \mathrm{M})$ for $24 \mathrm{~h}$, and then photographed with inverted contrast microscopy (100× magnification). Scale bars: $50 \mu \mathrm{m}$. (B) Representative images of colony formation assays using BCPAP and $8505 \mathrm{C}$ cells treated with $(0,1,5$ and $10 \mu \mathrm{M})$ for 8 days. ( $C$ and D) Quantification of the colony formation assays in BCPAP and 8505 C cells. Data are shown as mean \pm S.D. $(n \geq 3)$, 
Membranes were probed with primary antibodies. Bound primary antibodies were recognized by HRP-linked secondary antibodies (GE Healthcare). Immunoreactivity was detected by ECL Plus (Beyotime) and Kodak light film. Digital images of films were taken with BioRad Molecular Imager Gel Doc XR. Primary antibodies used for Western blot and immunohistochemistry are TP53 (Cell Signaling Technology), Cl-caspase 3 (Cell Signaling Technology), Cl-PARP (Cell Signaling Technology), TUBULIN (Huada), p-ERK (Cell Signaling Technology), ERK (Cell Signaling Technology), p-MEK (Cell Signaling Technology), MEK (Cell Signaling Technology), Ki67 (Abcam).

\section{RNA interference}

Small interfering RNAs (siRNA) against TP53 and control RNA were purchased from Sigma. The small siRNA target TP53 (EHU123221) was purchased from Sigma. All siRNA transfections were performed using Lipofectamine3000 Transfection Reagent (Invitrogen) following the manufacturer's recommendations. The final concentration of the siRNA molecules is $10 \mathrm{nM}$ and cells were treated with anlotinib $24 \mathrm{~h}$ later.

\section{Immunofluorescence assay}

Cells were fixed in $4 \%$ paraformaldehyde for $20 \mathrm{~min}$, and then permeabilized with $0.2 \%$ Triton $\mathrm{X}-100$ for $30 \mathrm{~min}$. After being blocked with $5 \%$ goat serum for $2 \mathrm{~h}$, cells were incubated with primary antibodies for $4-6 \mathrm{~h}$ at room temperature or overnight at $4^{\circ} \mathrm{C}$. Then cells were washed and incubated with secondary antibodies and/or rhodamine-phalloidin (Molecular Probe). Alexa Fluor 488 anti-mouse was used as secondary antibodies (Molecular Probe), and Hoechst 33342 (Sigma) for nuclei staining. Epifluorescent images were taken with Olympus IX81 microscope. Confocal images were captured using Leica TCS SP5 confocal microscope.

\section{Wound healing assay}

Cells were seeded into 6-well plate and grown to a confluent monolayer. A line was scratched on the cell monolayer with a p200 pipette tip. The plates were washed with PBS to remove detached cells, and then incubated with complete growth media containing $0,1,5$ or $10 \mu \mathrm{M}$ anlotinib for $12 \mathrm{~h}$. Cell migration was observed under a phase-contrast microscope at $40 \times$ magnification at 0 and $12 \mathrm{~h}$ post-induction of injury. Migration distance was analyzed using ImageJ software. Four pairs of images were analyzed for each sample.

\section{Colony formation assay}

For colony forming assay, cells were seeded in a 6-well plate at 300 cells per well in duplicates. Twenty-four hours later, cells were cultured with media containing $0,1,5$ or $10 \mu \mathrm{M}$ anlotinib for 8 days. Cells were then stained with $0.05 \%$ crystal violet before photographing.

\section{In vivo tumorigenicity assay}

All of the experimental protocols and animal care were approved by the Ethics Committee of the Tianjin Medical University Cancer Institute and Hospital, and were in compliance with the principles and procedures outlined in the NIH Guide for the Care and Use of Laboratory Animals. K1 cells were harvested and resuspended at $1 \times 10^{7} \mathrm{cell} / \mathrm{mL}$ with phosphate-buffered saline. Groups of 4-week-old male BALB/c athymic nude mice (each group, $n=5$ ) were subcutaneously injected at the buttock with $0.1 \mathrm{~mL}$ of the suspension. Tumor growth was measured on day 6 after injection, and then every 3 days. One group were intraperitoneally treated with $3 \mathrm{mg} / \mathrm{kg}$ anlotinib, once daily for two weeks. Another group were treated with equivoluminal DMSO, once daily for 2 weeks. At the indicated time points, the animals were killed and the tumors were excised and measured.

\section{Haematoxylin and eosin staining and immunohistochemistry (IHC)}

Dissected tumor tissues preserved in $4 \%$ PFA at $4^{\circ} \mathrm{C}$ for $24 \mathrm{~h}$, dehydrated through xylenes and alcohols, and embedded in paraffin. Sections were cut at $5 \mu \mathrm{m}$ and stained with haematoxylin and eosin (HE) for histological examination. Immunohistochemistry was performed according to standard protocols. Signal was visualized with DAB Substrate Kit (MaiXin Bio). Images were taken with Leica DM3000 Microscope.

\section{Statistical analysis}

All data were analyzed by Student's $t$-test. Statistically significant $P$ values were indicated in figures as follows: ${ }^{* *} P<0.001,{ }^{* *} P<0.01,{ }^{*} P<0.05$.

\section{Results}

\section{Anlotinib inhibits the proliferation of PTC and ATC cells}

To assess whether anlotinib inhibits the proliferation of cells derived from different thyroid tumor subtypes, we treated

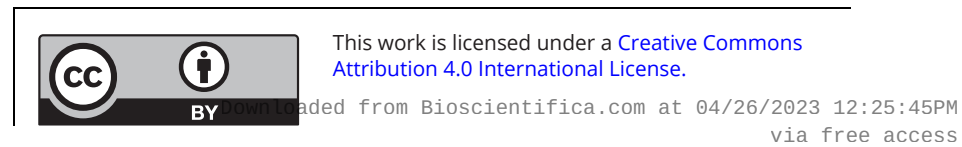


BCPAP (a PTC cell line) and 8505C (an ATC cell line) with anlotinib at increasing concentrations $(0,1,5$ and $10 \mu \mathrm{M})$ for $24 \mathrm{~h}$. The morphologic changes of thyroid cancer cells were examined under a phase-contrast microscope. The number of cells is dramatically reduced with the increasing concentration of anlotinib. Cells start to shrink, lose their normal shape, become round and ultimately detach from the culture dish (Fig. 1A). Next, we asked if the antitumor effect of anlotinib is ubiquitous for various thyroid cancer subtypes. Three PTC lines (BCPAP, K1 and IHH4) and three ATC lines (8505C, CAL-62 and BHT-101) were treated with anlotinib at different concentrations, and then subjected to the MTT assay. The viability of cells in all six thyroid cancer cell lines were all reduced when the concentration of anlotinib was greater than $1 \mu \mathrm{M}$ (Supplementary Fig. 1A, see section on supplementary data given at the end of this article). $\mathrm{IC}_{50}$ for all six cell lines range from $3.02 \mu \mathrm{M}$ to $5.42 \mu \mathrm{M}$ (Supplementary Fig. 1B). These data suggest that anlotinib effectively inhibits the cell viability of both PTC and ATC cells. Overall, PTC cells are slightly more sensitive to anlotinib than ATC cells.

To further validate the anti-proliferative effects of anlotinib on thyroid cancer cells, colony formation assay was carried out with BCPAP and 8505C cells. Twenty-four hours after seeding, BCPAP and $8505 \mathrm{C}$ cells were treated with anlotinib at various concentrations $(0,1,5$ and $10 \mu \mathrm{M})$ for 8 days. The number and the size of colonies were measured on day 9. As the concentration of anlotinib increases, the number of colonies decreases, and the size of colonies becomes smaller (Fig. 1B, C and D). These data confirm that anlotinib elicits an anti-proliferative effect on BCPAP and 8505C cells in a dose-dependent manner.

\section{Anlotinib induces G2/M arrest and abnormal spindle assembly in thyroid carcinoma cells}

Given the anti-proliferative effect of anlotinib on thyroid cancer cells, we asked whether anlotinib inhibits cell proliferation by regulating cell cycle progression. BCPAP and $8505 \mathrm{C}$ cells were treated with or without $10 \mu \mathrm{M}$ anlotinib for $24 \mathrm{~h}$. The cells were then stained with PI and analyzed by flow cytometry. The fraction of G2/M cells increases markedly in anlotinib-treated BCPAP and $8505 \mathrm{C}$ cells. In addition, anlotinib treatment also leads to a slight enhancement of sub-G1 fraction, indicating that anlotinib induces cell apoptosis as well (Fig. 2).

The activation of the BRAF/MEK/ERK pathway plays an important role in thyroid cancer (Espinosa et al. 2007, Girotti \& Marais 2013). The BRAFV600E mutation is found in approximately one-half of PTC patients and one-fourth of ATC cases and is associated poor prognosis (Li et al. 2012, Liu et al. 2016, Xing et al. 2013). We first tested whether anlotinib inhibits cell proliferation and induces $\mathrm{G} 2 / \mathrm{M}$ arrest by blocking the BRAF/MEK/ERK signaling pathway. To evaluate the effect of anlotinib on the BRAF/MEK/ERK pathway in BCPAP and 8505C cells, the phosphorylation levels of MEK and ERK were determined by Western blot. Only at high concentrations (10 and $15 \mu \mathrm{M}$ ), anlotinib reduces the phosphorylation levels of MEK and ERK in BCPAP cells (Supplementary Fig. 2A).
A

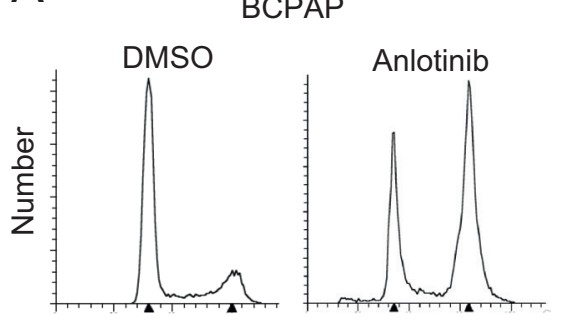

$\mathrm{Pl}$

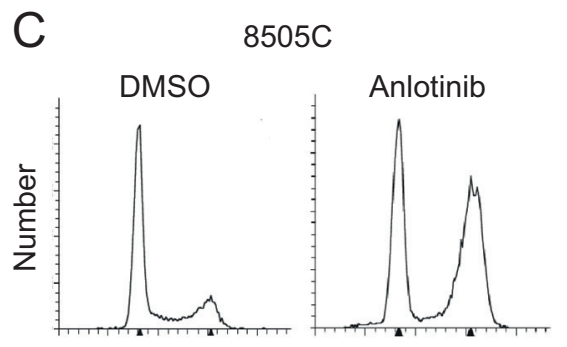

PI
B

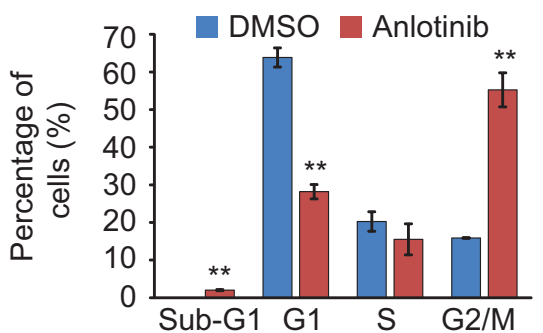

D

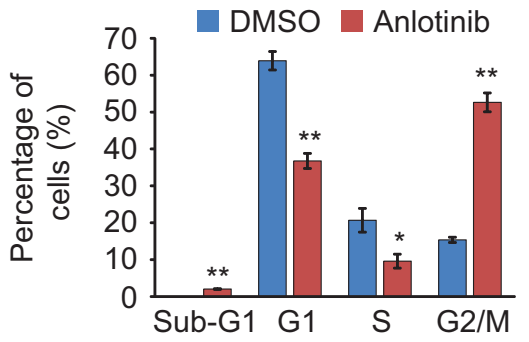

\section{Figure 2}

Anlotinib induces $\mathrm{G} 2 / \mathrm{M}$ phase arrest in thyroid cancer cells. (A and C) BCPAP cells (A) and 8505C cells (C) were treated with DMSO or $10 \mu \mathrm{M}$ anlotinib for $24 \mathrm{~h}$ and stained with PI. Cell cycle distribution was assessed with flow cytometry and quantified by Modifit software. (B and D) The percentage of cells in sub-G1, G1, S and G2/M phase were calculated and plotted. Data are shown as mean \pm s.D. $(n \geq 3)$. 
A

Anlotinib $(\mu \mathrm{M}): 0$
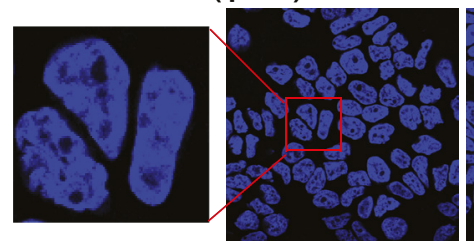

\section{BCPAP}

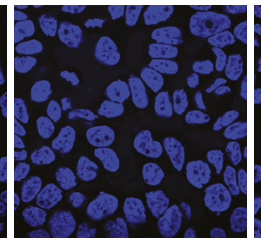

5

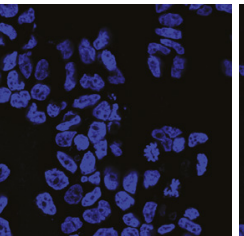

10

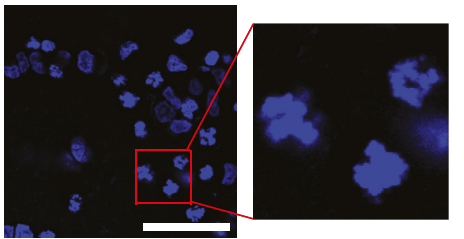

B
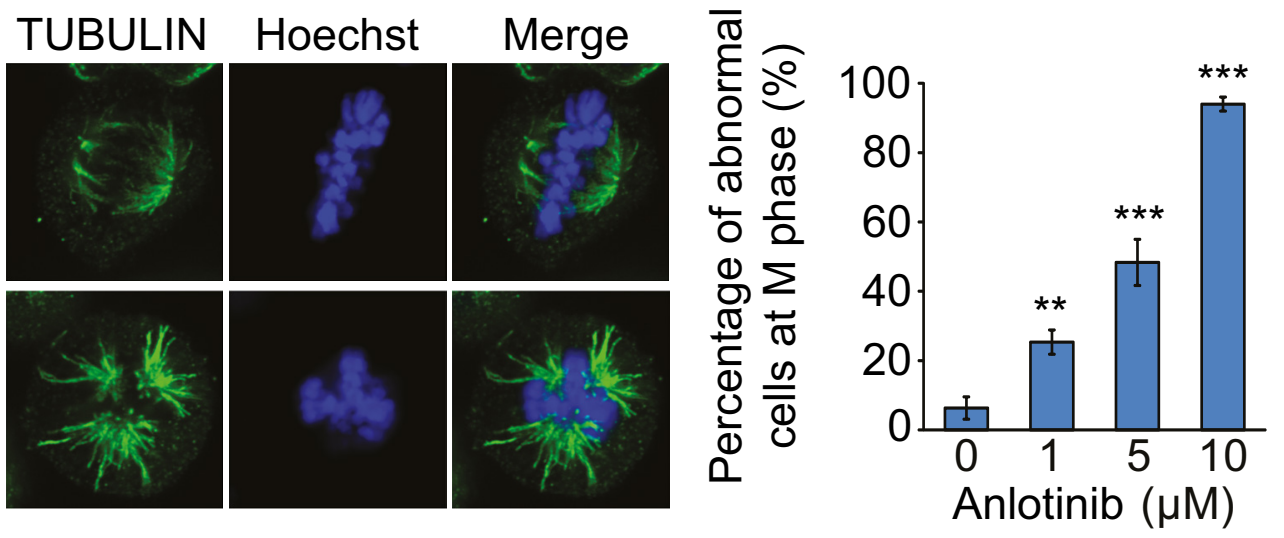

C
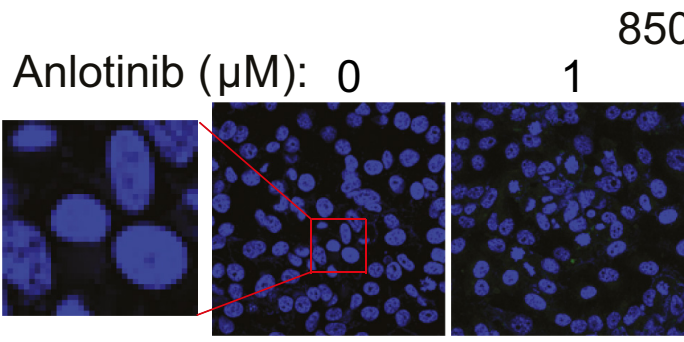

$8505 \mathrm{C}$
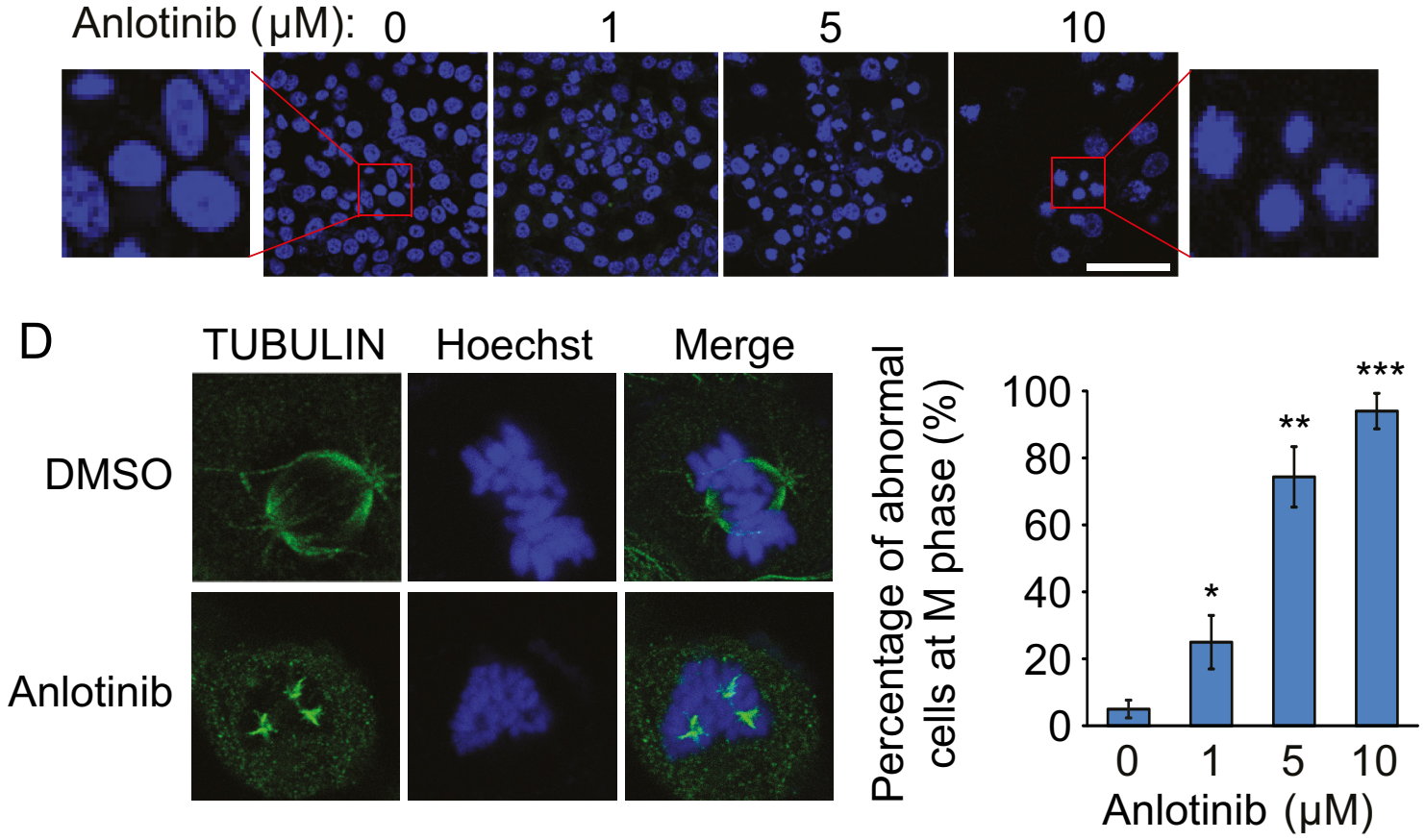

Figure 3

Anlotinib leads to abnormal spindle formation in thyroid cancer cells. (A and C) BCPAP cells (A) and 8505C cells (C) were treated with anlotinib (0, 1, 5 and $10 \mu \mathrm{M}$ ) for $24 \mathrm{~h}$. The morphological changes of nuclei were examined using Hoechst 33342 staining. Scale bars: $100 \mu \mathrm{m}$. The blowup images from the red boxes show nuclear condensation and structural changes after exposure to anlotinib. (B and D) Effect of anlotinib on mitotic spindle formation in thyroid cancer cells. Cells were treated with or without $10 \mu \mathrm{M}$ anlotinib for $24 \mathrm{~h}$ and subjected to immunofluorescence staining for TUBULIN. The right plots show the percentage of cells with abnormal spindle in the metaphase cells treated with $0,1,5$ and $10 \mu \mathrm{M}$ anlotinib. Data are shown as mean \pm s.D. ( $n \geq 3$ ). 
A

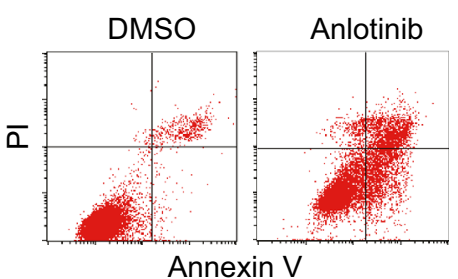

B

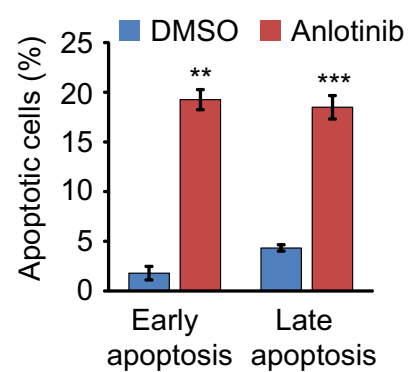

C

Anlotinib

$(\mu \mathrm{M})$ :

TP53

$\mathrm{Cl}$-caspase 3

CI-PARP

TUBULIN
BCPAP

Annexin V

apoptosis apoptosis

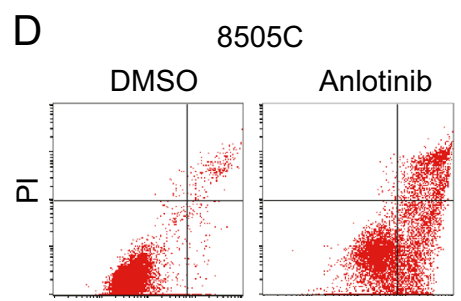

Annexin V

E

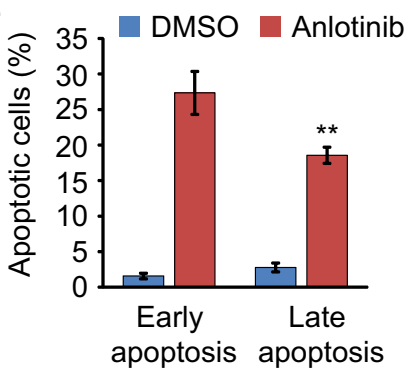

F

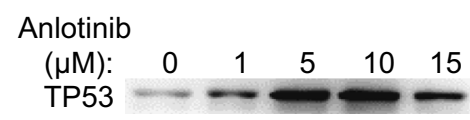

Cl-caspase 3

CI-PARP

TUBULIN

\section{Figure 4}

Anlotinib induces apoptosis in thyroid cancer cells. (A and D) BCPAP (A) and 8505C (D) cells were treated with DMSO and $10 \mu \mathrm{M}$ anlotinib for $36 \mathrm{~h}$. Cells were stained with Annexin V-FITC/PI and analyzed by flow cytometry. The low right (LR) quadrant of the histograms indicates the early apoptotic cells, and the upper right quadrant indicates the late apoptotic cells. (B and E) Quantification results of experiments described in A and D. Data are shown as mean \pm s.D. $(n \geq 3)$. (C and F) BCPAP (C) and 8505C (F) cells were treated with anlotinib $(0,1,5,10$ and $15 \mu \mathrm{M})$ for $36 \mathrm{~h}$. Cell lysates were prepared and subjected to Western blot to detect the expression of TP53, cleaved caspase 3 (CL-caspase 3 ) and cleaved PARP (CL-PARP).
In contrast, MEK and ERK phosphorylation in $8505 \mathrm{C}$ cells is not affected by anlotinib, even at the highest concentration tested (Supplementary Fig. 2B), suggesting that the anti-proliferative effect of anlotinib is not mediated by the suppression of the BRAF/MEK/ERK pathway, at least in $8505 \mathrm{C}$ cells. Moreover, a specific MEK inhibitor PD0325901 (PD), which effectively blocks the MEK/ERK pathway, has little effect on the cell viability of BCPAP and 8505C cells (Supplementary Fig. 2). The above data showed that the anti-proliferative effect of anlotinib on thyroid cancer cells is not through inactivating the BRAF/MEK/ERK pathway.

To address how anlotinib arrests cells at the G2/M phase, we checked the morphology of nuclei in BCPAP and $8505 \mathrm{C}$ cells with or without anlotinib treatment for 24h. A significant fraction of nuclei in anlotinib-treated cells become condensed and fragmented, while the control cells without anlotinib treatment exhibit normal intact nuclei (Fig. 3A and C). Further examination of the spindle microtubule organization in mitotic cells revealed that anlotinib causes abnormal spindle organization. Rather than di-polar spindles, tri-polar spindles were observed in anlotinib-treated mitotic cells. The percentage of mitotic cells with abnormal spindles increases in an anlotinib dose-dependent manner (Fig. 3B and D). These data suggested that deregulated spindle formation by anlotinib treatment might account for the G2/M phase arrest of thyroid cancer cells.

\section{Anlotinib induces apoptosis in thyroid cancer cells partly through activating the TP53 pathway}

We have observed anlotinib-induced thyroid cancer cell apoptosis in cell cycle analysis (Fig. 2). To further confirm the apoptosis induced by anlotinib, BCPAP and $8505 \mathrm{C}$ cells, with or without anlotinib treatment for $36 \mathrm{~h}$, were stained with Annexin V-FITC/PI and analyzed by flow cytometry. The results demonstrated that anlotinib treatment induces apoptosis in both BCPAP and 8050C cells. The fractions of early and late apoptotic anlotinibtreated BCPAP cells are 19.3 and 19.2\%, respectively. And 27.3 and $18.6 \%$ of anlotinib-treated $8050 \mathrm{C}$ cells are in the early and late apoptosis, respectively (Fig. 4A, B, $\mathrm{D}$ and $\mathrm{E})$. These results further validated that anlotinib stimulates the apoptosis of thyroid cancer cells.

In order to investigate the mechanism how anlotinib induces apoptosis in thyroid cancer cells, the expression levels of apoptosis-related proteins, including TP53, 
A

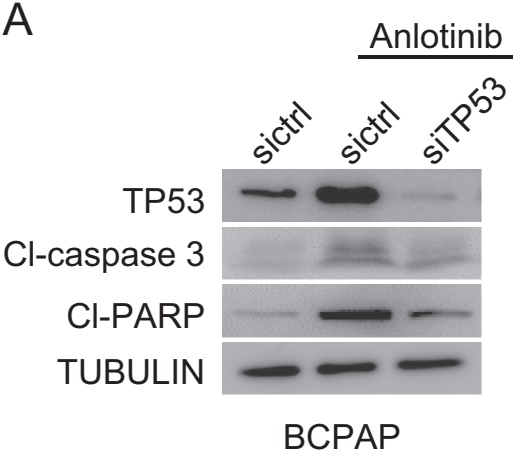

B

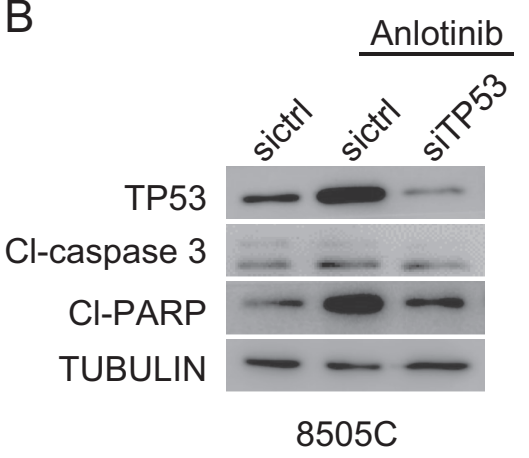

C

Anlotinib
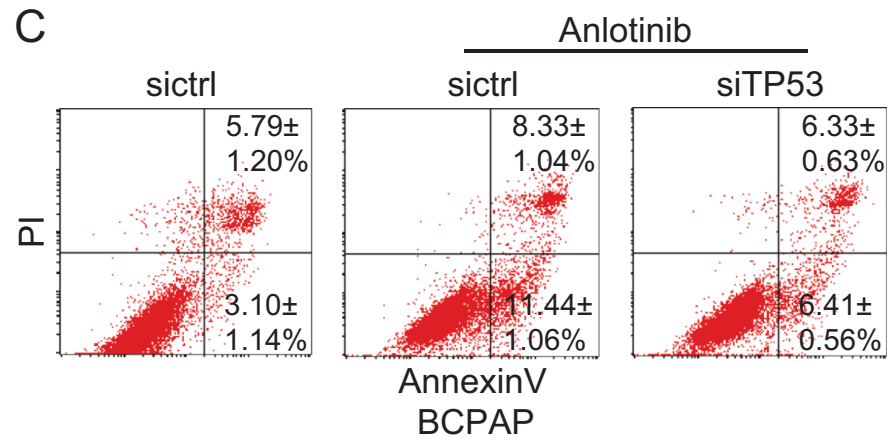

$\mathrm{D}$

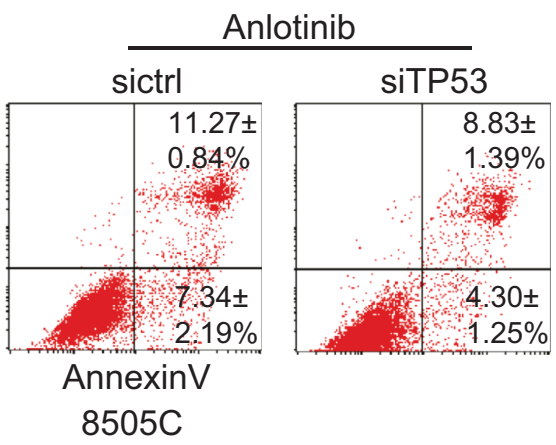

\section{Figure 5}

Anlotinib induces apoptosis partly through activating the TP53 pathway. (A and B) siRNA target TP53 and control RNA were transfected into thyroid cancer cells. Twenty-four hours after transfection, the cells were treated with or without $10 \mu \mathrm{M}$ anlotinib for $24 \mathrm{~h}$. Cell lysates were prepared and subjected to Western blot. (C and D) Thyroid cancer cells described in (A and B) were stained with Annexin V-FITC/PI and analyzed with flow cytometry. Data are shown as mean \pm S.D. $(n \geq 3)$. cleaved caspase 3 and cleaved PARP, were examined by Western blot. The results revealed that anlotinib upregulates the expression of TP53, as well as apoptotic markers cleaved caspase 3 and cleaved PARP, in a dosedependent manner (Fig. 4C and F). Therefore, anlotinib might induce the apoptosis of thyroid cancer cells through activating the TP53 pathway.

To confirm the role of TP53 in the apoptosis induced by anlotinib, TP53 was knocked down by siRNA in thyroid cancer cells. After transfection with the control RNA and siRNA, the cells were treated with $10 \mu \mathrm{M}$ anlotinib for $24 \mathrm{~h}$. Compared with the control cells transfected with siRNA, the levels of apoptotic markers cleaved caspase 3 and cleaved PARP in TP53-knockdown thyroid cancer cells are reduced (Fig. 5A and B). Consistently, few TP53knockdown thyroid cancer cells undergo apoptosis upon anlotinib treatment (Fig. 5C and D). These data suggested that the TP53 pathway at least partly, if not entirely, mediates the apoptosis inducing effect of anlotinib.

\section{Anlotinib inhibits the migration of thyroid cancer cells}

To investigate whether anlotinib impairs the migration of thyroid cancer cells, we performed wound-healing assay. We found that anlotinib dose dependently decreased the migration ability of BCPAP and 8505C cells. The wound closure rates of anlotinib-treated cells were lower than those of non-treated cells (Fig. 6A and B).

Since cell migration depends on actin filament (F-actin) remodeling, we tested the hypothesis that anlotinib inhibits thyroid cancer cell migration by 
A
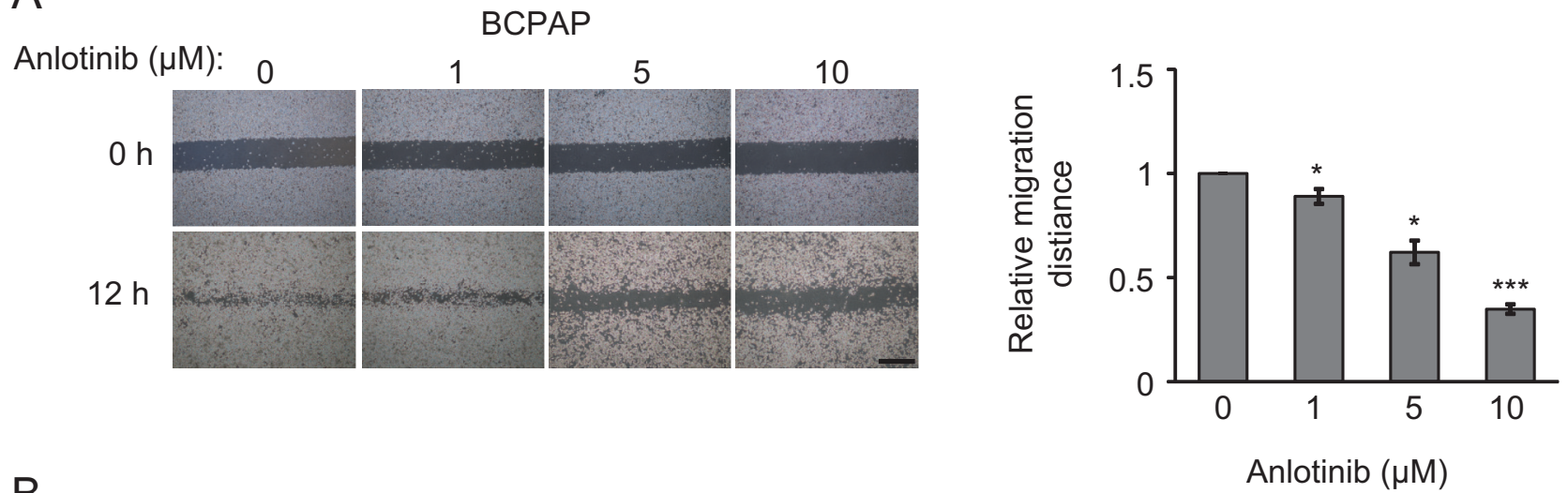

B

Anlotinib $(\mu \mathrm{M})$

$8505 \mathrm{C}$

\section{$\mathrm{Oh}$}

$12 \mathrm{~h}$
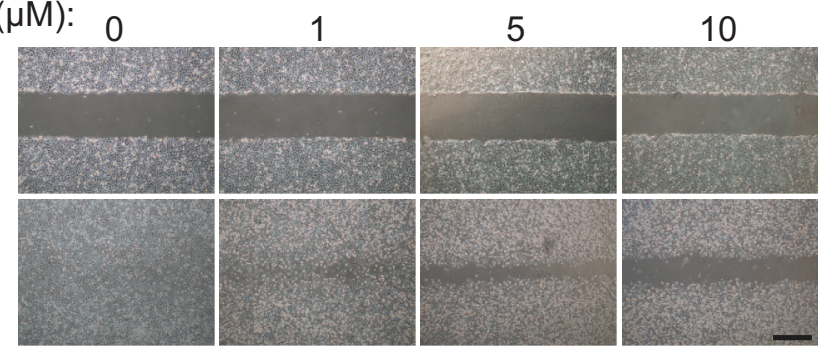

C

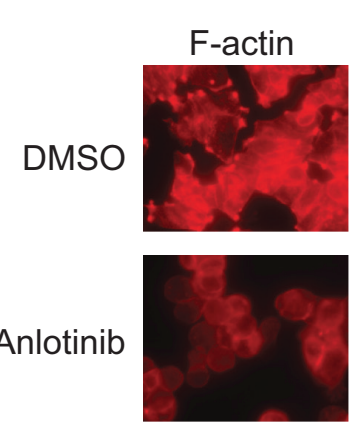

BCPAP
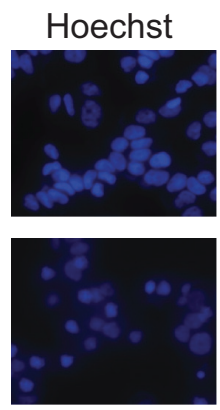
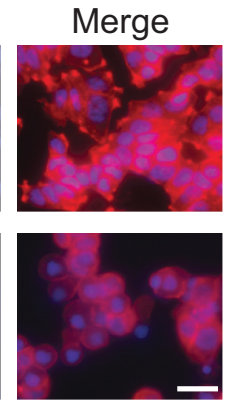

D

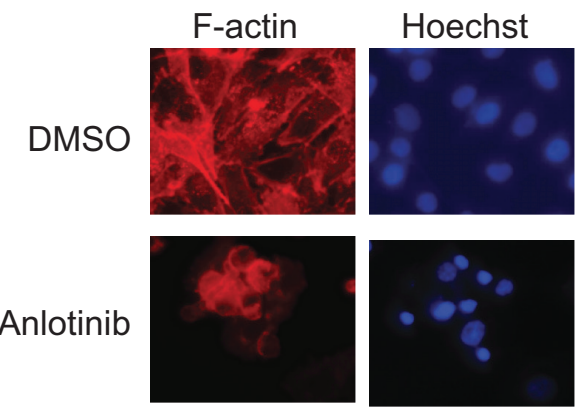

$8505 \mathrm{C}$
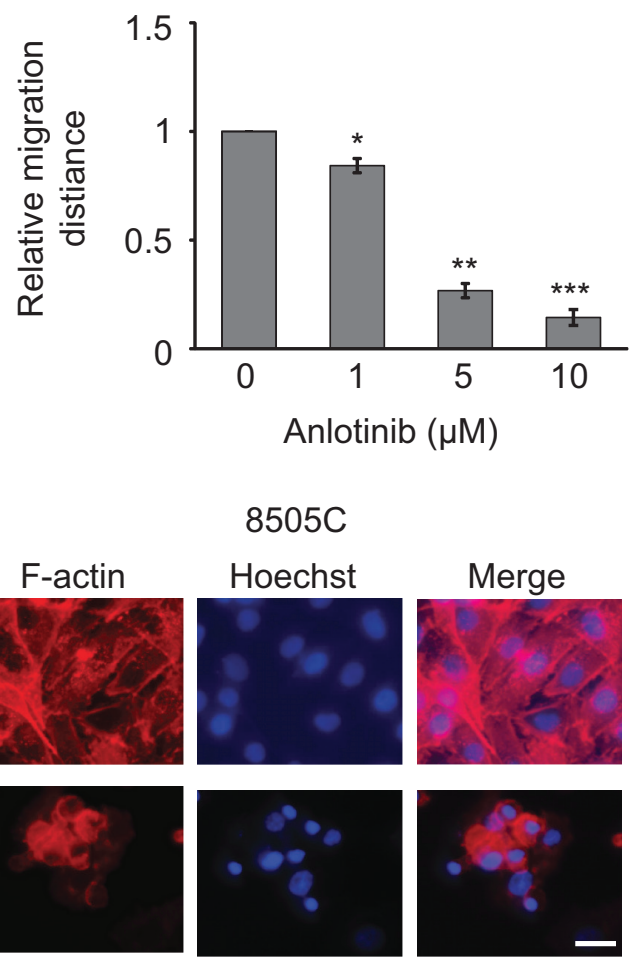

\section{Figure 6}

The effects of anlotinib on the migration of thyroid cancer cells. (A and B) Representative images of wound-healing assays using BCPAP (A) and 8505C (B) cells in the presence of 0,1,5 and $10 \mu \mathrm{M}$ anlotinib. Scale bars: $25 \mu \mathrm{m}$. The plots on the right show the quantification of wound healing assays. Data are shown as mean \pm S.D. $(n \geq 3)$. (C and D) The expression and cellular distribution of F-actin in BCPAP (C) and 8505C cells (D). Cells were treated with DMSO or $10 \mu \mathrm{M}$ anlotinib for $24 \mathrm{~h}$, and then stained with rhodamine-phalloidin (red) for F-actin. Hoechst 33342 was applied to counter-stain the nucleus (blue). Scale bars: $100 \mu \mathrm{m}$.

interfering F-actin organization. F-actin was stained with rhodamine-phalloidin in both BCPAP and 8505C cells with or without anlotinib treatment. It is notable that anlotinib treatment alters the organization of F-actin in BCPAP and 8505C cells. Control untreated cells are elongated, with one or two predominant lamellipodia per cell. In anlotinib-treated cells, less F-actin is formed, and the lamellipodia nearly disappeared (Fig. 6C and
D). These data suggested that anlotinib might interfere F-actin formation to suppress cell migration.

\section{Anlotinib suppresses thyroid tumor growth in vivo}

Next, we used a K1 xenograft tumor model to determine whether anlotinib inhibits thyroid cancer growth in vivo. The anlotinib-treated group started to exhibit 
A

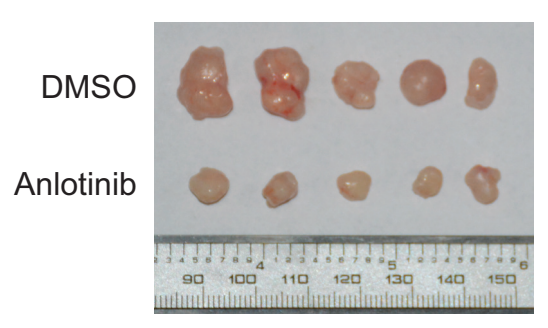

C

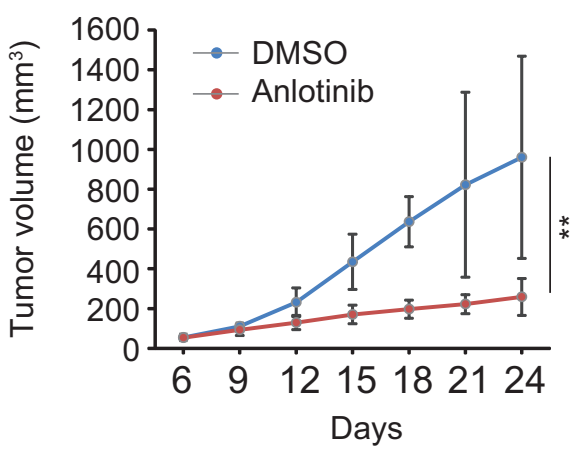

B

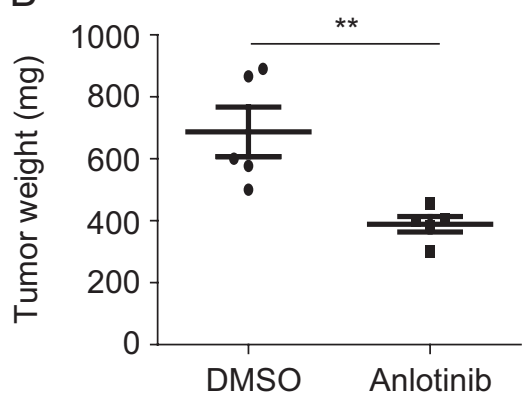

D

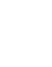

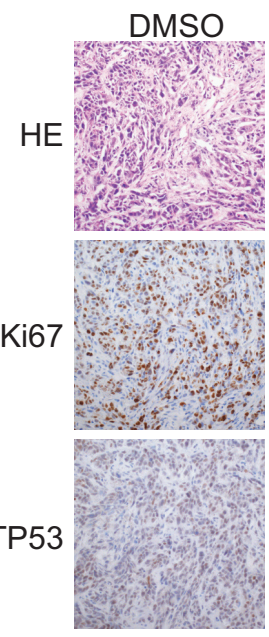

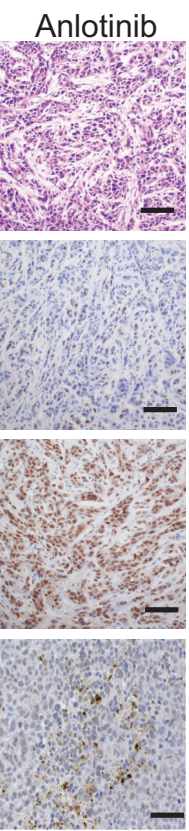

\section{Figure 7}

Anlotinib suppresses tumor growth in vivo. (A) Images of dissected tumors from nude mice injected with K1 cells of DMSO treated group $(n=5)$ and anlotinib treated group $(n=5)$. (B) Tumor weights of control and anlotinib-treated groups. (C) Tumor volumes with or without anlotinib treatment. (D) HE staining, Ki67, TP53 and CL-caspase $3 \mathrm{IHC}$ staining of the tumors with or without anlotinib treatment. Scale bars: $100 \mu \mathrm{m}$. reduced tumor growth on day 9 and showed significantly decreased tumor weight and tumor volume at the end of the experiment (Fig. 7A, B and C). Moreover, anlotinib had little effect on bodyweight in mice during the course of the experiment (Supplementary Fig. 3). Compared with control group, anlotinib-treated group had fewer Ki67-positive cells, meaning fewer proliferating cells (Fig. 7D). Consistent with cell-based assay (Figs 4 and 5), anlotinib treatment also elevates the expression level of TP53 and cleaved caspase 3 in thyroid cancer xenografts (Fig. 7D), implying that apoptosis might be induced in these anlotinib-treated thyroid cancer xenografts. These data demonstrated that anlotinib inhibits thyroid tumor growth in vivo.

\section{Discussion}

In most well-differentiated thyroid cancer cases, patients have excellent prognosis, whereas a minority of well-differentiated thyroid cancer patients die due to locoregional recurrence and distant metastasis (Zhang et al. 2016). However, ATC is extremely aggressive, and the median survival of ATC patients is only 3-5 months after diagnosis (Machens et al. 2005). The development of novel effective therapeutic agents for thyroid cancer, particularly for ATC, remains an urgent medical requirement. In this study, we investigated the potential therapeutic effect of a novel multi-kinase inhibitor anlotinib on thyroid cancers. Our data suggested that anlotinib has antitumor effect for both PTC and ATC cells. The $\mathrm{IC}_{50}$ values of anlotinib for the six PTC or ATC cell lines used in the present study range from $3.02 \mu \mathrm{M}$ to $5.42 \mu \mathrm{M}$. Moreover, daily intraperitoneal treatment of $3 \mathrm{mg} / \mathrm{kg}$ anlotinib significantly suppressed tumor growth in the thyroid cancer xenograft model. These data provide the foundation of dosage for future clinical trials.

Several mechanisms may contribute to the antitumor effect of anlotinib. First, anlotinib treatment compromises the proper organization of mitotic spindle, leading to G2/M arrest and a slower proliferation rate. Second, anlotinib 
elevates the expression of TP53, thus inducing cell apoptosis. Third, anlotinib prevents cell migration, likely through interfering F-actin formation.

Interestingly, both BCPAP and 8505C cells harbor TP53 mutations, D259Y and R248G, respectively (Saiselet et al. 2012). Nevertheless, TP53 still mediates the proapoptotic effect of anlotinib in these two cell lines. There are at least two possible reasons for this phenomenon. D259Y and R248G mutations affect the DNA-binding activity of TP53, thus resulting in loss of function of TP53. Nevertheless, these mutations are different from null TP53 mutation. The DNA-binding ability of D259Y and R248G TP53 mutants can be reactivated by a compound, named TP53 reactivation and induction of massive apoptosis (PRIMA-1) (Rehman et al. 2005, Messina et al. 2012). Thus, anlotinib might be able to reactivate D259Y and R248G TP53 mutants. Alternatively, the transcriptionindependent function of TP53 might account for its proapoptotic effect in D259Y and R248G TP53-mutant cells. Overexpression of a mutant TP53, lacking most of the DNA-binding domain and completely deficient in transactivation function, could trigger apoptosis (Haupt et al. 1995, Green \& Kroemer 2009). Similarly, activation of TP53 was found to trigger apoptosis even in the absence of a nucleus (Chipuk et al. 2003). Thus, D259Y and R248G TP53 mutants are able to induce apoptosis through its transcription-independent function.

However, the direct target(s) of anlotinib mediating its therapeutic effect on thyroid cancers remain elusive. Despite its important role in the pathogenesis of thyroid cancer (Murugan et al. 2009), the BRAF/MEK/ERK signaling pathway is unlikely the key downstream target of anlotinib. First, anlotinib suppresses the BRAF/MEK/ ERK pathway only in BCPAP cells, but not in 8050C cells, while anlotinib effectively inhibits the proliferation of both cell lines. Second, inactivation of the BRAF/MEK/ ERK pathway by a MEK inhibitor PD0325901 slows down the proliferation of BCPAP slightly, but not 8050C cells (Supplementary Fig. 2). Future efforts are required to identify the direct target(s) of anlotinib responsible for its anti-proliferation, pro-apoptosis and anti-migration effects. With this knowledge, drug with better specificity and less side-effect may be developed.

In summary, our data demonstrated that anlotinib exerts antitumor effects on thyroid cancer cells through inhibition of cell growth, induction of apoptosis and suppression of cell migration. Importantly, anlotinib is effective against various thyroid carcinoma cell lines, including both PTC and ATC. Importantly, anlotinib also suppresses thyroid tumor growth in vivo. Thus, anlotinib could be developed as a novel effective antitumor agent for thyroid cancer treatment, especially for poorly differentiated papillary thyroid cancers and ATC, for which no effective therapeutic treatment is available.

\section{Supplementary data}

This is linked to the online version of the paper at https://doi.org/10.1530/ ERC-17-0558.

\section{Declaration of interest}

The authors declare that there is no conflict of interest that could be perceived as prejudicing the impartiality of the research reported.

\section{Funding}

This work did not receive any specific grant from any funding agency in the public, commercial, or not-for-profit sector.

\section{Author contribution statement}

$X R, X S, Q D, Y Y, X H, X W$ and X S performed the experiments, M G and L C conceived the experiments and wrote the manuscript.

\section{Acknowledgements}

This work was supported by the National Natural Science Foundation of China (Grant No. 81472580, 81272282, 81502322, and 31271547) and the Natural Science Foundation of Tianjin, China (No. 13JCQNJC10200 and 14JCYBJC23600).

\section{References}

Baudin E \& Schlumberger M 2007 New therapeutic approaches for metastatic thyroid carcinoma. Lancet Oncology 8 148-156. (https:// doi.org/10.1016/s1470-2045(07)70034-7)

Chen W, Zheng R, Baade P D, Zhang S, Zeng H, Bray F, Jemal A, Yu XQ \& He J 2016 Cancer statistics in China 2015. CA: A Cancer Journal for Clinicians 66 115-132. (https://doi.org/10.3322/caac.21338)

Cho BY, Choi HS, Park YJ, Lim JA, Ahn HY, Lee EK, Kim KW, Yi KH, Chung JK,Youn YK, et al. 2013 Changes in the clinicopathological characteristics and outcomes of thyroid cancer in Korea over the past four decades. Thyroid 23 797-804. (https://doi.org/10.1089/ thy.2012.0329)

Covell LL \& Ganti AK 2015 Treatment of advanced thyroid cancer: role of molecularly targeted therapies. Targeted Oncology 10 311-324. (https://doi.org/10.1007/s11523-014-0331-z)

Chipuk JE, Maurer U, Green DR \& Schuler M 2003 Pharmacologic activation of p53 elicits Bax-dependent apoptosis in the absence of transcription, Cancer Cell 4 371-381. (https://doi.org/10.1016/S15356108(03)00272-1)

Enewold L, Zhu K, Ron E, Marrogi AJ, Stojadinovic A, Peoples GE \& Devesa SS 2009 Rising thyroid cancer incidence in the United States by demographic and tumor characteristics, 1980-2005. Cancer Epidemiology, Biomarkers and Prevention 18 784-791. (https://doi. org/10.1158/1055-9965.EPI-08-0960)

Espinosa AV, Porchia L \& Ringel MD 2007 Targeting BRAF in thyroid cancer. British Journal of Cancer 96 16-20. (https://doi.org/10.1038/ sj.bjc.6603520) 
Fagin JA \& Mitsiades N 2008 Molecular pathology of thyroid cancer: diagnostic and clinical implications. Best Practice and Research Clinical Endocrinology and Metabolism 22 955-969. (https://doi.org/10.1016/j. beem.2008.09.017)

Girotti MR \& Marais R 2013 Deja Vu: EGF receptors drive resistance to BRAF inhibitors. Cancer Discovery 3 487-490. (https://doi. org/10.1158/2159-8290.CD-13-0131)

Green DR \& Kroemer G 2009 Cytoplasmic functions of the tumour suppressor p53. Nature 458 1127-1130. (https://doi.org/10.1038/ nature07986)

Haupt Y, Rowan S, Shaulian E, Vousden KH \& Oren M 1995 Iduction of apoptosis in Hela cells by trans-activation-deficient p53. Genes and Development 9 2170-2183. (https://doi.org/10.1101/ gad.9.17.2170)

Kilfoy BA, Zheng T, Holford TR, Han X, Ward MH, Sjodin A, Zhang Y, Bai Y, Zhu C, Guo GL, et al. 2009 International patterns and trends in thyroid cancer incidence, 1973-2002. Cancer Causes Control 20 525-531. (https://doi.org/10.1007/s10552-008-9260-4)

Kondo T, Ezzat S \& Asa SL 2006 Pathogenetic mechanisms in thyroid follicular-cell neoplasia. Nature Reviews Cancer 6 292-306. (https:// doi.org/10.1038/nrc1836)

Li C, Lee KC, Schneider EB \& Zeiger MA 2012 BRAF V600E mutation and its association with clinicopathological features of papillary thyroid cancer: a meta-analysis. Journal of Clinical Endocrinology and Metabolism 97 4559-4570. (https://doi.org/10.1210/jc.2012-2104)

Liu C, Chen T \& Liu Z 2016 Associations between BRAF(V600E) and prognostic factors and poor outcomes in papillary thyroid carcinoma: a meta-analysis. World Journal of Surgical Oncology 14241. (https://doi.org/10.1186/s12957-016-0979-1)

Lorusso L, Pieruzzi L, Biagini A, Sabini E, Valerio L, Giani C, Passannanti P, Pontillo-Contillo B, Battaglia V, Mazzeo S, et al. 2016 Lenvatinib and other tyrosine kinase inhibitors for the treatment of radioiodine refractory, advanced, and progressive thyroid cancer. OncoTargets Therapy 9 6467-6477. (https://doi.org/10.2147/OTT. S84625)

Machens A, Holzhausen H-J \& Dralle H 2005 The prognostic value of primary tumor size in papillary and follicular thyroid carcinoma. Cancer 103 2269-2273. (https://doi.org/10.1002/cncr.21055)

McFarland D \& Misiukiewicz K 2014 Sorafenib in radioactive iodinerefractory well-differentiated metastatic thyroid cancer. OncoTargets and Therapy 7 1291. (https://doi.org/10.2147/ott.s49430)

Murugan AK, Dong J, Xie J \& Xing M 2009 MEK1 mutations, but not ERK2 mutations, occur in melanomas and colon carcinomas, but none in thyroid carcinomas. Cell Cycle 8 2122-2124. (https://doi. org/10.4161/cc.8.13.8710)

Messina RL, Sanfilippo M, Vella V, Pandini G, Vigneri P, Nicolosi ML, Giani F, Vigneri R \& Frasca F 2012 Reactivation of p53 mutants by prima-1 (corrected) in thyroid cancer cells. International Journal of Cancer 130 2259-2270. (https://doi.org/10.1002/ijc.26228)

Nagaiah G, Hossain A, Mooney CJ, Parmentier J \& Remick SC 2011 Anaplastic thyroid cancer: a review of epidemiology, pathogenesis, and treatment. Journal of Oncology 2011 542358. (https://doi. org/10.1155/2011/542358)

Rehman A, Chahal MS, Tang X, Bruce JE, Pommier Y \& Daoud SS 2005 Proteomic identification of heat shock protein 90 as a candidate target for p53 mutation reactivation by PRIMA-1 in breast cancer cells. Breast Cancer Research 7 R765-R774. (https://doi.org/10.1186/ bcr1290)

Sherman SI 2009 Advances in chemotherapy of differentiated epithelial and medullary thyroid cancers. Journal of Clinical Endocrinology and Metabolism 94 1493-1499. (https://doi.org/10.1210/jc.2008-0923)

Smallridge RC, Marlow LA \& Copland JA 2009 Anaplastic thyroid cancer: molecular pathogenesis and emerging therapies. EndocrineRelated Cancer 16 17-44. (https://doi.org/10.1677/ERC-08-0154)

Smallridge RC, Ain KB, Asa SL, Bible KC, Brierley JD, Burman KD, Kebebew E, Lee NY, Nikiforov YE, Rosenthal MS, et al. 2012 American Thyroid Association guidelines for management of patients with anaplastic thyroid cancer. Thyroid 22 1104-1139. (https://doi.org/10.1089/thy.2012.0302)

Sun Y, Niu W, Du F, Du C, Li S, Wang J, Li L, Wang F, Hao Y, Li C, et al. 2016 Safety, pharmacokinetics, and antitumor properties of anlotinib, an oral multi-target tyrosine kinase inhibitor, in patients with advanced refractory solid tumors. Journal of Hematology and Oncology 9 105. (https://doi.org/10.1186/s13045-016-0332-8)

Saiselet M, Floor S, Tarabichi M, Dom G, Hebrant A, van Staveren WC \& Maenhaut C 2012 Thyroid cancer cell lines: an overview. Frontiers in Endocrinology 3 133. (https://doi.org/10.3389/fendo.2012.00133)

Wang C, Chen J, Cao W, Sun L, Sun H \& Liu Y 2016 Aurora-B and HDAC synergistically regulate survival and proliferation of lymphoma cell via AKT, mTOR and Notch pathways. European Journal of Pharmacology 779 1-7. (https://doi.org/10.1016/j. ejphar.2015.11.049)

Xing M 2013 Molecular pathogenesis and mechanisms of thyroid cancer. Nature Reviews Cancer 13 184-199. (https://doi.org/10.1038/ nrc3431)

Xing M, Alzahrani AS, Carson KA, Viola D, Elisei R, Bendlova B, Yip L, Mian C, Vianello F, Tuttle RM, et al. 2013 Association between BRAF V600E mutation and mortality in patients with papillary thyroid cancer. JAMA 309 1493. (https://doi.org/10.1001/jama.2013.3190)

Zhang L, Liu L, He X, Shen Y, Liu X, Wei J, Yu F \& Tian J 2016 CHIP promotes thyroid cancer proliferation via activation of the MAPK and AKT pathways. Biochemical and Biophysical Research Communications 477 356-362. (https://doi.org/10.1016/j. bbrc.2016.06.101)

Received in final form 5 July 2018

Accepted 21 August 2018

Accepted Preprint published online 23 August 2018 https://erc.bioscientifica.com https://doi.org/10.1530/ERC-17-0558
(C) 2019 The authors Published by Bioscientifica Ltd. Printed in Great Britain
This work is licensed under a Creative Commons Attribution 4.0 International License.

ded from Bioscientifica.com at $04 / 26 / 2023$ 12:25:45PM 